Proyecciones

Vol. $11 \mathrm{~N}^{0} 1$, pp.01-09 Mayo 1992

Universidad Católica del Norte

Antofagasta - Chile

\title{
SPECTRA OF RELATED GRAPHS AND
} SELF-REPRODUCING POLYHEDRA*

EDUARDO MONTENEGRO AND REINALDO SALAZAR

Universidad Católica de Valparaíso, Valparaiso, Chile.

\section{DAVID L. POWERS}

Clarkson University, New York, USA

\begin{abstract}
If $G$ is a d-valent graph, the eigenvalues of the adjacency matrix of $G$ determine those of the line graph, the subdivision graph and the graph made by replacing vertices with complete graphs. A property of the eigenvectors of the graphs of certain regular polyhedra is then seen to carry over to some truncations of the polyhedra.
\end{abstract}

* This work was supported by the research project FONDECYT 89-0164, Substitution of vectices by Graphs 


\section{Spectra of related graphs.}

Let $G$ be a connected graphs (loops and multiple edges not allowed) with $p$ vertices and $q$ edges. (Definitions of graph-theoretic terms may be found in [2].) We will be particulary interested in forming other graphs from $G$. The first is the line graph $G_{L}$ of $G$, whose vertices are the edges of $G$. Two vertices of $G_{L}$ are adjacent if and only if the corresponding edges of $G$ have a common endpoint. The second is the subdivision $G_{S}$ of $G$, which is formed by inserting a new vertex in each edge of G. (See Figure 1.)

A graph $G$ with $p$ vertices and $q$ edges can be described by a $p \times q$ incidence matrix $R$, which has a 1 in the $i, k$-position if vertex $i$ is an endpoint of edge $k$, or a 0 if not. The same graph can also be described by a $p \times p$ adjancency matriz $A$, which has a 1 in the $i, j$-position if vertices $i$ and $j$ are the endpoints of an edge, or a 0 if not.

The relations among incidence and adjacency matrices of a graph $G$, its line graph $G_{L}$ and its subdivision graph $G_{S}$ are as follows [4,p.16]

$$
\begin{aligned}
& R R^{T}=A_{G}+D \\
& R^{T} R=A_{L}+2 I \\
& {\left[\begin{array}{cc}
0 & R \\
R^{T} & 0
\end{array}\right]=A_{S}}
\end{aligned}
$$

In Eq. (1.1), $D$ is the degree matriz, $D=\operatorname{diag}\left\{d_{1}, \ldots, d_{p}\right\}$, where $d_{i}$ is the degree or valency of vertex $i$.

Generally, there is no connection between the eigenvalues of $A_{G}$ and $A_{L}$. However, if $G$ is $d$-valent, then $D=d I$, and we will assume this to be true. Now, let $x, y$ be a pair of singular vectors of $R$ corresponding to singular value $\rho>0$; that is $R^{T} x=\rho y$ and $R y=\rho x$. Then

$$
\begin{aligned}
& R R^{T} x=\rho^{2} x=\left(A_{G}+d I\right) x \\
& R^{T} R y=\rho^{2} y=\left(A_{L}+2 I\right) y \\
& {\left[\begin{array}{cc}
0 & R \\
R^{T} & 0
\end{array}\right]\left[\begin{array}{ll} 
& x \\
\pm & y
\end{array}\right]= \pm \rho\left[\begin{array}{ll} 
& x \\
\pm & y
\end{array}\right]}
\end{aligned}
$$


From these equations we see that the eigenvalues of $G, G_{L}$ and $G_{S}$ are related by

$$
\lambda_{L}=\lambda_{G}+d-2
$$

$$
\lambda_{S}= \pm \sqrt{\lambda_{G}+d}
$$

and that the multiplicity of any eigenvalue $\lambda_{G}>-d$ carries over to the related $\lambda_{L}$ or $\lambda_{S}$.

It is known [4, p. 114] that the rank of $R$ is $p-1$ if $G$ is bipartite or $p$ otherwise. In the bipartite case, there are $q-p+1$ independent solutions of $R y=0$ and one of $R^{T} x=0$. Thus, for a bipartite $G$, we have eigenvalues $\lambda_{G}=-d$ (simple), $\lambda_{L}=-2$ (multiplicity $q-p+1$ ), and $\lambda_{S}=0$ (multiplicity $q-p+2)$. For a nonbipartite graph $G,-d$ is not an eigenvalue of $A_{G}$, but $\lambda_{L}=-2$ (multipliocity $q-p$ ) and $\lambda_{S}=0$ (multiplicity $q-p$ ). (Note that with $d \geq 2, q=p d / 2 \geq p$.)

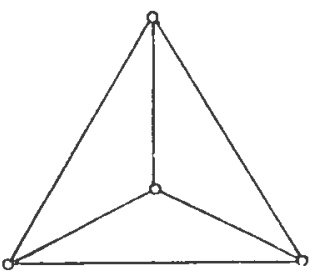

G

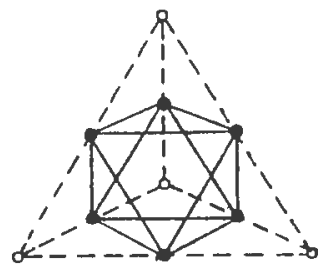

$\mathrm{G}_{\text {L }}$

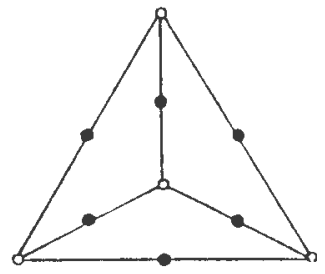

$\mathrm{G}_{\mathrm{S}}$

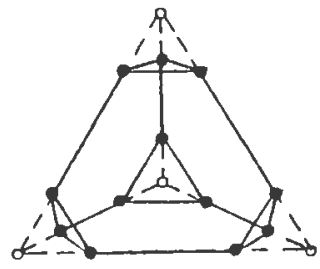

$G_{k}$

Figure 1 
We are interested also in the graph $G_{K}$ (See Figure 1) made from $G$ by replacing each vertex $v$ with a complete graph $K_{d}$, so that each of the $d$ edges of $G$ that were incident with $v$ is made incident to exactly one vertex of $K_{d}$. (This is a special case of a graph operation called substitution. See [7].) If we start from a $d$-valent graph $G$ with $p$ vertices, then $G_{K}$ is $d$-valent with $p d$ vertices. The key to finding the spectrum of $G_{K}$ lies in recognizing that $G_{K}$ is the line graph of the subdivision graph of $G$.

Theorem 1. Let $G$ be a $d$-valent graph and $G_{K}$ the made by replacing each vertex of $G$ by the complete graph $K_{d}$. The eigenvalues $\lambda_{K}$ of the adjacency matrix of $G_{K}$ are related to those of $G$ by

$$
\lambda_{K}=\frac{1}{2}\left(d-2 \pm \sqrt{d^{2}+4+4 \lambda_{G}}\right)
$$

Proof: The adjacency matrix of $A_{S}$ is given in Eq.(1.3). Suppose that $Q$ is an incidence matrix of the subdivision graph $G_{S}$. Then

$$
Q Q^{T}=A_{S}+D_{S}=\left[\begin{array}{cc}
d I & R \\
R^{T} & 2 I
\end{array}\right]
$$

Note that $G_{S}$ is not generally $d$-valent: each of the new vertices introduced in the subdivision process has degree 2. The adjacency matrix of the line graph of $G_{S}$ is $A_{K}=Q^{T} Q-2 I$. The eigenvalues other than -2 of $A_{K}$ coincide (including multiplicity) with those of

$$
M=Q Q^{T}-2 I=\left[\begin{array}{cc}
(d-2) I & R \\
R^{T} & 0
\end{array}\right] .
$$

Now, suppose that $x$ and $y$ are singular vectors of $R$ corresponding to singular value $\rho>0$. Consider the partitioned matrix product

$$
M\left[\begin{array}{ll}
x & 0 \\
0 & y
\end{array}\right]=\left[\begin{array}{cc}
(d-2) x & \rho x \\
p y & 0
\end{array}\right]=\left[\begin{array}{ll}
x & 0 \\
0 & y
\end{array}\right]\left[\begin{array}{cc}
d-2 & \rho \\
\rho & 0
\end{array}\right]
$$

Since both $x$ and $y$ are nonzero, the eigenvalues of the last $2 \times 2$ matrix are eigenvalues of $M$ and hence of $A_{K}$. It may be verified easily that they satisfy the relation given in the statement of the theorem.

The graph $G_{S}$ is always bipartite, and so its line graph $G_{K}$ has -2 as an eigenvalue of multiplicity $q_{s}-p_{s}+1=p d / 2-p+1$. One occurence of the eigenvalue $\lambda_{K}=-2$ is included in Eq.(1.6), for $\lambda_{G}=d$. 
Example. Let $G$ be the complete graph on 4 vertices as shown in Figure 1. The eigenvalues, with multiplicities in brackets, are shown in the Table. Note that 0 [2] was added to the spectrum of $G_{S},-2[2]$ to that of $G_{L_{1}}$ and -2[4] to that if $G_{K}$.

\section{TABLE}

$\begin{array}{lll}\text { graph } & \text { \#vertices } & \text { Eigenvalues } \\ & & \\ G \cong K_{4} & 4 & 3[1],-1[3] \\ G_{S} & 10 & \sqrt{6}[1], 2[3], 0[2],-2[3],-\sqrt{6}[1] \\ G_{L} \cong K_{2,2,2} & 6 & 4[1], 0[3],-2[2] \\ G_{K} & 12 & 3[1], 2[3],-1[3],-2[5]\end{array}$

\section{Truncations of polyhedra.}

Let $P$ be an $n$-dimensional polytope with $p$ vertices. The skeleton of $P$ is the graph formed by its vertices and edges. For example, Figure 1 shows the skeleton of a tetrahedron. Godsil [5] suggested that a graph $G$ on $p$ vertices could produce a polytope in the following way. Let $\lambda$ be an eigenvalue of the adjacency matrix of A with multiplicity $m$, and let $U$ be a $p \times m$, matrix of orthonormal eigenvectors:

$$
A U=\lambda U, U^{T} U=1
$$

The distinct rows of $U$ can be interpreted as the coordinates of points in $m$ dimensional euclidean space. The convex hull of these points, $C(\lambda)$, is the polytope associated with the eigenvalue $\lambda$.

If a polytope $P$ is isomorphic to a polytope $C(\lambda)$ associated with an eigenvalue of its skeleton, we say that $P$ is self-reproducing [6]. This means that the mere adjacency information in the skeleton can generate the metric information in the coordinates of the vertices of the polytope. To prove that an $n$-dimensional polytope is self-reproducing, one must show (1.1) that the adjacency matrix of the skeleton has an eigenvalue $\lambda$ of multiplicity $n$, and (1.2) that the sum of the vectors of position of the neighbors of vertex $i$ is $\lambda$ times the vector of position of vertex $i$, for each $i$. This second requirement is equivalent to the ith row of the eigenvector equation (2.1).

For example, the skeleton of the cube has eigenvalues $3[1], 1[3],-1[3],-3[1]$. We may take the vertices to be $( \pm 1, \pm 1, \pm 1)$. The three neighbors of vertex $(a, b, c)$ are $(-a, b, c),(a,-b, c),(a, b,-c)$, and $(-a, b, c)+(a,-b, c)+(a, b,-c)=1(a, b, c)$. Thus, we have shown that the cube, being isomorphic to $C(1)$, is self-reproducing. 
In [6] it was shown that many regular polytopes are self-reproducing, including all polygons and the platonic solids. In all cases, the polytope of the second largest eigenvalue is isomorphic to the original polytope. We will show that the same properties apply to some truncations of these polytopes.

For simplicity we consider only 3-dimensional polytopes (polyhedra). A simple truncation [3, p. 148] of a polyhedron $P$ is formed by cutting off each vertex with a plane through the midpoints of the edges issuing from it. For example the octahedron is the simple truncation of a tetrahedron, and the cuboctahedron is the simple truncation of both the octahedron and the cube

The skeleton of the truncation of $P$ has as vertices the midpoints of the edges. The midpoints surrounding a vertex of $P$ are joined in a cycle, which is the vertex figure of the vertices of $P$. Thus, if the skeleton of $P$ is a 3 -valent graph, the skeleton of the truncation of $P$ is isomorphic to the line graph of the skeleton of $P$. Hence, we can use Eq. (1.4) to find the spectrum of these truncations.

Theorem 2. The simple truncations of the platonic solids are self-reproducing.

Proof. Let $P$ be a platonic solid with a 3 -valent skeleton $G$, and let $\lambda_{G}$ be the second largest eigenvalue of $G$. Then, if $U$ is the $p \times 3$ matrix of coordinates of vertices of $G$, we know [6] that $A_{G} U=\lambda_{G} U$. Let $R$ be an incidence matrix so that $R R^{T}=A_{G}+3 I$, and set $V=\frac{1}{2} R^{T} U$. Then each row of $V$ contains the coordinates of the midpoints of an edge of $P$, and

$$
\begin{aligned}
A_{L} V & =\left(R^{T} R-2 I\right) V \\
& =\left(\lambda_{G}+1\right) V
\end{aligned}
$$

Since $\lambda_{G}+1$ is an eigenvalue of $A_{L}$ with the same multiplicity, 3 , that $\lambda_{G}$ has in the spectrum of $A_{G}$, we have shown that the truncation of $P$ is selfreproducing.

Finally, we note that cube and octahedron have the same truncation (cuboctahedron), and that icosahedron and dodecahedron also have the same truncation (icosidodecahedron).

An intermediate truncation $[3$, p. $148 ; 1$, p. 76$]$ of a polyhedron is made by cutting off each vertex with a plane that intercepts each of the edges issuing the vertex at a point between the vertex and the midpoint of the edge. For a polyhedron with 3-valent skeleton $G$, the skeleton of an intermediate truncation is isomorphic to $G_{K}$ 
Theorem 3. Let $P$ be a polyhedron whose skeleton is 3-valent. If $P$ is selfreproducing, then there is an intermediate truncation of $P$ that is also selfreproducing.

Proof: Let the coordinates of a vertex of $P$ and its three neighbors be row matrices $u_{0}, u_{1}, u_{2}, u_{3}$. On each line segment from $u_{0}$ to $u_{i}$, we introduce two points with coordinates

$$
\begin{aligned}
& w_{0 i}=(1-t) u_{0}+t u_{i}, \\
& w_{i 0}=(1-t) u_{i}+t u_{0},
\end{aligned}
$$

with $t<\frac{1}{2}$. These are vertices of an intermediate truncation of $P$. We are given that

$$
u_{1}+u_{2}+u_{3}=\lambda u_{0}
$$

and we wish to show that

$$
w_{i, 0}+w_{0, i+1}+w_{0, i+2}=\alpha w_{0 i}
$$

for some $t$ and $\alpha$, and for each $i$. (Subscripts $i, i+1, i+2$ are interpreted modulo 3 , in the set $\{1,2,3\}$.)

Substitution of Eqs. (2.2) and (2.3) into Eq. (2.5) leads to the equation

$$
(t+(1-t)(2-\alpha)) u_{0}+(1-t-\alpha t) u_{i}+t u_{i+1}+t u_{i+2}=0
$$

Eq. (2.4) permits us to eliminate $u_{i+1}$ and $u_{i+2}$ leaving

$$
(t(1+\lambda)+1-t)(2-\alpha)) u_{0}+(1-2 t-\alpha t) u_{1}=0 .
$$

Since $u_{0}$ and $u_{1}$ are independent row matrices, their coefficients in the equation above must both be 0 . These can be reduced to

$$
\alpha^{2}-\alpha-(3+\lambda)=0
$$




$$
t=\frac{1}{\alpha+2}
$$

Note that de roots of $\mathrm{Eq}$. (2.6) satisfy Eq. (1.6), confirming the fact that $\alpha$ is an eigenvalue of $G_{K}$. Only the larger of the roots of Eq. (2.6) gives a value of $t<\frac{1}{2}$.

Since we showed in Theorem 1 that eigenvalue multiplicities carry over from $G$ to $G_{K}$, and since $P$ is self-reproducing, both $\lambda$ and $\alpha$ must have multiplicity 3 .

It is interesting to note we have not used any symmetry of the polyhedron $P$ in the proof, except the symmetry in Eq. (2.4). Since this symmetry is inherited by the intermediate truncation, it seems that the process could be continued indefinitely.

\section{Conclusion.}

We have shown that the eigenvalue of $G_{K}$ can be obtained from those $G$, if $G$ id $d$-valent. As an application, we proved that the simple and intermediate truncations of some self-reproducing polyhedra are also self-reproducing.

\section{REFERENCES}

[1] Brondsted, A.: An Introduction to Convex Polytopes. Springer, New York, 1983.

[2] Chartrand, G. and Lesniak, L.: Graphs \& Digraphs, second ed. Wadsworth. \& Brooks/Cole, Monterey, CA., 1979.

[3] Coxeter, H.S.M.: Regular Polytopes, third ed. Dover, New York, 1973.

[4] Cvetkovic, D.M.; Doob, M. and Sachs, H.: Spectra of Graphs. VEB, Berlin/Academic Press, New York, 1980.

[5] Godsil, G.: Graphs, groups and polytopes. In Combinatorial Mathematics VI(Canberra 1977), D.A. Holton and J. Seberry, eds. Springer, New York, pp. 157-164, 1978.

[6] Licata, C. and Powers, D.L.: A surprising property of some regular polytopes. Scientia, 1, pp 73-80, 1988.

[7] Montenegro, E.: A result on the order and size of graphs that represent a finite group. Extracta Mathematicae, 2, pp 14-16, 1987. 
Recibido : 5 de Agosto de 1991.

David L. Powers

Department of Mathematics \& Computer Science

Clarkson University

Postsdam, New York 13699 USA.

Eduardo Montenegro and Reinaldo Salazar

Instituto de Matemáticas

Universidad Católica de Valparaíso

Casilla 4059, Valparaíso, Chile. 